\title{
Timing of Subsequent Fractures after an Initial Fracture
}

\author{
Tineke A. C. M. van Geel • Kirsten M. B. Huntjens • \\ Joop P. W. van den Bergh • Geert-Jan Dinant • \\ Piet P. Geusens
}

Published online: 18 June 2010

(C) The Author(s) 2010. This article is published with open access at Springerlink.com

\begin{abstract}
A prior fracture is a well-documented risk factor for a subsequent fracture and it doubles the risk of subsequent fractures. Few studies have investigated the time that elapses between the initial and subsequent fracture. These studies show that the subsequent fracture risk is not constant, but fluctuates over time. The risk of subsequent vertebral, hip, and nonvertebral non-hip fractures is highest immediately after initial hip, clinical, and radiographic vertebral fractures and nonvertebral fractures
\end{abstract}

T. A. C. M. van Geel $(\bowtie) \cdot$ G.-J. Dinant

Department of General Practice, Maastricht University/Caphri, P.O. Box 616, 6200 MD Maastricht, The Netherlands

e-mail: t.vangeel@hag.unimaas.nl

G.-J. Dinant

e-mail: geertjan.dinant@hag.unimaas.nl

\section{K. M. B. Huntjens}

Department of General Surgery and Trauma Surgery,

Maastricht University Medical Centre/Caphri,

P.O. Box 5800, 6202 AZ Maastricht, The Netherlands

e-mail: k.huntjens@ah.unimaas.nl

J. P. W. van den Bergh

Department of Internal Medicine,

VieCuri Medical Centre Noord-Limburg,

Tegelseweg 210,

5912 BL Venlo, The Netherlands

e-mail: jvdbergh@viecuri.nl

\section{J. P. W. van den Bergh}

Department of Internal Medicine,

Maastricht University Medical Centre/Nutrim,

P.O. Box 5800, 6202 AZ Maastricht, The Netherlands

\section{P. P. Geusens}

Subdivision Rheumatology, Department of Internal Medicine,

Maastricht University Medical Centre/Caphri,

P.O. Box 5800, 6202 AZ Maastricht, The Netherlands

e-mail: piet.geusens@scarlet.be and declines afterward, regardless of gender, age, and initial fracture location. These studies indicate the need for early action after an initial fracture with medical interventions that have an effect within a short term to reduce the preventable risks of subsequent fractures.

Keywords Osteoporosis · Fractures · Elderly

\section{Introduction}

In terms of fractures, osteoporosis is one of the leading health problems in the Western world according to the World Health Organization and is a growing problem due to the aging population [1, 2]. However, in terms of bone mineral density (BMD) T-score less than -2.5 , osteoporosis is only one of the many risk factors for fractures.

A prior fracture is yet another well-known risk factor for a subsequent fracture, independent of BMD [3]. The risk of a subsequent fracture is doubled in the presence of a prior fracture (pooled relative risk [RR], 2.0) [3]. However, several studies have shown that the risk of subsequent fracture is not constant, but fluctuates over time, being highest immediately after the initial fracture $[4 \cdot \bullet, 5 \cdot \bullet, 6-8]$.

One of the earliest studies on the timing between first and subsequent fractures focused on men and women who had been hospitalized because of a clinical vertebral fracture and in whom the risk of all subsequent fractures was highest within the first year. Lindsay et al. [8] found that within 1 year after an incident radiographic vertebral fracture, $20 \%$ of patients had a new radiographic vertebral fracture.

These observations had a growing impact on further research on timing of subsequent fractures in relation to a first fracture, and focused on all fractures or specific initial 
or subsequent fracture locations. If subsequent fractures cluster in time, this has as a clinical consequence the need for immediate attention for fracture patients to decrease the risk of subsequent fracture. Furthermore, if fractures cluster in time, algorithms for case findings should take into account the variable risk of subsequent fracture over time.

Several studies have studied the time relation between fractures. However, the time relation between fractures has been reported in several ways in terms of RR, absolute risk (AR), as percentages of all subsequent fractures that occurred over short-and long-term follow-up, and as survival or cumulative curves. First and subsequent fractures were studied at variable fracture locations (vertebral, nonvertebral, major, minor, and hip fractures). We reviewed these aspects in the literature on studies that reported the time relation between fractures and, as far as reported, review the RR, AR, and percent of subsequent fractures according to baseline and subsequent fracture location.

\section{Clustering in Time of Fractures after a Vertebral Fracture}

Morphometric vertebral fractures are the most frequent osteoporosis-related fractures, but only around one-third come to clinical attention with the classical signs and symptoms of an acute fracture. Therefore, studies on vertebral fractures differ in including only clinical vertebral fractures or in systematic performance of radiographs of the thoracic and lumbar spine.

\section{Repeat Morphometric Vertebral Fractures}

Only one study is available on repeat radiographic vertebral fractures. It was performed by analyzing placebo-treated patients $(n=2,725)$ pooled from several fracture studies with risedronate [8]. These women had prevalent vertebral fractures $[9,10]$, low femoral neck BMD, or risk factors for hip fracture [11]. All subjects received calcium supplementation $(1,000 \mathrm{mg} / \mathrm{d})$. Women with serum 25 -hydroxyvitamin D levels of less than $16 \mathrm{ng} / \mathrm{mL}(40 \mathrm{nmol} / \mathrm{L})$ at baseline also received vitamin D supplementation (up to $500 \mathrm{IU} / \mathrm{d}$ ).

In this study, the 1-year incidence of radiographic vertebral fractures could be analyzed in three groups of patients: 1) patients without a prevalent fracture; 2) patients with a prevalent radiographic vertebral fracture before the start of the follow-up, and of which the timing of the previous radiographic vertebral fracture was unknown; and 3)patients who developed a new radiographic vertebral fracture during the study and who were followed yearly for the development of subsequent radiographic vertebral fractures.
The cumulative incidence of new radiographic vertebral fractures in the first year of the study follow-up (ie, the AR for new radiographic vertebral fractures in patients with and without previous radiographic vertebral fracture of unknown timing) was $6.6 \%$ in the total group (Fig. 1) [8].

In patients with of one or more radiographic vertebral fractures at baseline, in whom the timing of fractures was unknown, the risk of sustaining a radiographic vertebral fracture increased by fivefold during the initial year of the study compared with the incidence in subjects without prevalent radiographic vertebral fractures at baseline (RR, 5.1; 95\% CI, 3.1-8.4; $P<0.001)$. The 1-year AR for a first radiographic vertebral fracture was $1.9 \%, 4.6 \%$ in the presence of one vertebral fracture of unknown radiographic vertebral fracture and $9.9 \%$ and $12.5 \%$ in the presence of more than one or two radiographic vertebral fractures of unknown timing at baseline, respectively [8].

Among the 381 participants who developed an incident radiographic vertebral fracture during the study follow-up, the incidence of a new radiographic vertebral fracture in the subsequent year was $19.2 \%$ (95\% CI, 13.6-24.8) [8]. In summary, this study indicated that the 1-year AR for subsequent radiographic vertebral fractures is $1.9 \%$ for a first radiographic vertebral fracture, $4.6 \%$ in the presence of a radiographic vertebral fracture of unknown timing, and $19.2 \%$ within 1 year after a radiographic vertebral fracture had occurred. This study clearly showed that the risk of repeat radiographic vertebral fractures is highest during the first year after a radiographic vertebral fracture.

\section{Fractures after Clinical Vertebral Fractures}

A study focused on the magnitude and time course of the increase in risk of further fractures during a mean follow-up of 2.4 years (maximum, 8 years) in patients who were hospitalized for vertebral fracture $(n=28,869)$ [7]. It has been shown that there was a marked increase in subsequent

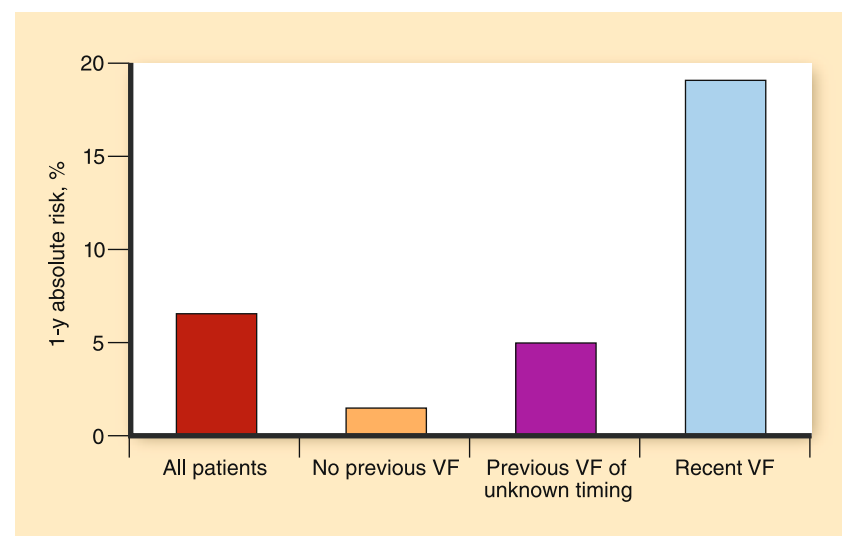

Fig. 1 One-year absolute risk (AR) for repeat radiographic vertebral fractures $(\mathrm{VFs})$ 
incidence of hip and all fractures within the first year following hospitalization for vertebral fracture in both men and women. Thereafter, fracture incidence declined toward, but did not attain baseline risk. The increase in fracture risk was more marked following low-energy vertebral fracture than following high-energy trauma. The RR for an incident fracture (compared with the general population) was at any age higher at 6 months (3.9-48.7, according to age) than at 4 years (1.4-15.2, according to age), was higher in men (5.6-48.7, according to age) than in women (3.9-32.0, according to age), and was higher in younger than in older patients (Fig. 2). The AR, expressed as the incidence of all subsequent fractures, was higher at 6 months $(7.8 \%$ to $21.5 \%$, according to age) than at 4 years $(1.9 \%$ to $10.2 \%$, according to age) and higher in women $(8.0 \%$ to $21.5 \%$, according to age) than in men ( $7.8 \%$ to $16.0 \%$, according to age) [7]. The timing of the percentage of fracture according to time of follow-up was not mentioned.

\section{Clustering in Time of Fractures after a Hip Fracture}

A total of 799 patients were followed during a mean follow-up of 22 years after their initial hip fracture. After the initial hip fracture, $27 \%$ of all subsequent fractures (mean number of subsequent fractures per patient: 2.3, range 1-11 fractures/patient) occurred within 2 years and $73 \%$ within 5 years [12]. The AR over 22 years was $45 \%$ in the total group and $86 \%$ in survivors. This indicates that the 2-year AR was about $12 \%$ in the total group and about $23 \%$ in survivors, and that the 5-year AR was $33 \%$ and $63 \%$, respectively [12].

A study in elderly living in a nursing home $(n=184)$ showed the same trend. The AR of the subsequent fractures was $12 \%$ within 1 year and $21 \%$ within 5 years, indicating that more than half of subsequent fractures during 5-year follow-up occurred within 1 year after a hip fracture [13].

In a study that included more than 170,000 men and women with an initial hip fracture, almost 28,000 patients sustained a subsequent hip fracture during 25 -year follow-up (mean follow-up, 3.8 years). After a first hip fracture, the cumulative incidence of subsequent hip fracture was $9 \%$ within 1 year and $20 \%$ within 5 years, compared with $2 \%$ and $12 \%$, respectively, in subjects without a hip fracture. Thus, nearly half of all subsequent hip fractures during 5-year follow-up occurred within 1 year. The RR for a second hip fracture was 11.8 at 1 month, decreasing exponentially to 3.0 at 6 months and 2.2 at 1 year, and did not normalize until after
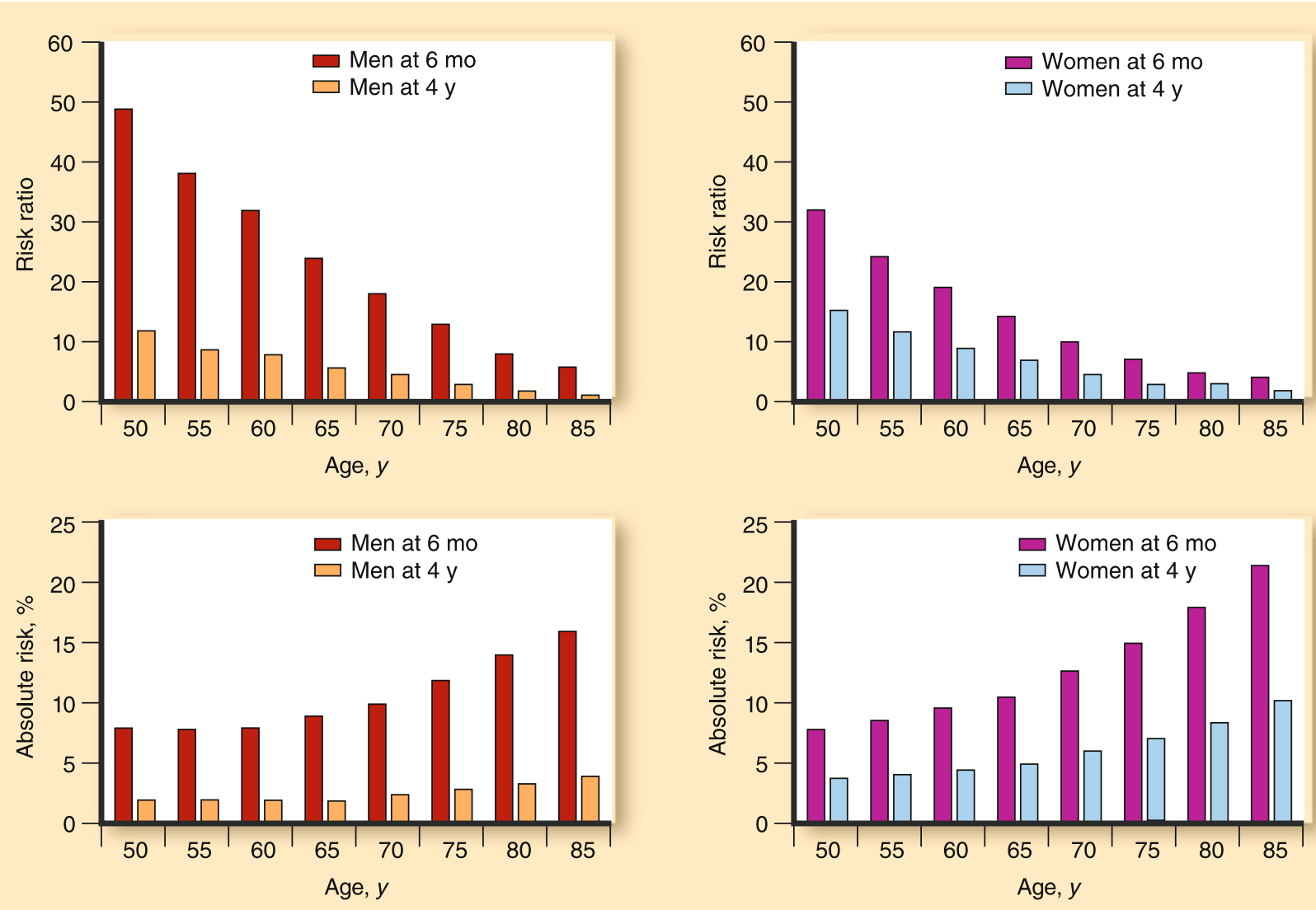

Fig. 2 Risk ratio and absolute risk of subsequent fracture 
15 years [14••]. Risk factors for a second hip fracture were female sex, age, alcoholism, any prior fracture, living alone, and having a higher income $[14 \bullet \bullet]$.

Similar results were reported in other studies $[15,16]$. In addition, almost all fractures after the initial hip fracture were caused by low-energy traumatic accidents (eg, fall from standing height or less, fall from a chair, or fall out of bed) $[12,13 \bullet, 14 \bullet \bullet, 15,16]$.

\section{Clustering in Time after Clinical Fractures}

One study focused on the risk of hip, forearm, clinical vertebral, or shoulder fractures in women and men between 60 and 80 years of age from the time they had a hip, clinical vertebral, or shoulder fracture in both outpatients and hospitalized patients [6]. Of all subsequent hip, clinical vertebral, forearm, or shoulder fractures during a follow-up of 5 years, 34\% occurred within the first year after the initial fracture (range, $31 \%$ to $45 \%$ ) and progressively decreased to $9 \%$ during the 5 th year (Fig. 3) [6].

We published a population-based study focusing on all clinical fractures (including high-energy or low-energy trauma fractures) in postmenopausal women [5••]. During a follow-up of 20 years (mean, 9 years), $23 \%$ of all subsequent fractures were sustained within 1 year after the initial fracture and $54 \%$ within 5 years. After 15 years, the subsequent fracture risk was no longer higher than the initial fracture risk $[5 \bullet \cdot]$.

In the Dubbo Osteoporosis Epidemiology Study in Australia, in community-dwelling men and women older than 60 years of age, the AR of subsequent low-energy trauma fracture was similar for men and women [4••]. About $41 \%$ of the subsequent fractures in women and $52 \%$ in men occurred within 2 years after the initial fracture

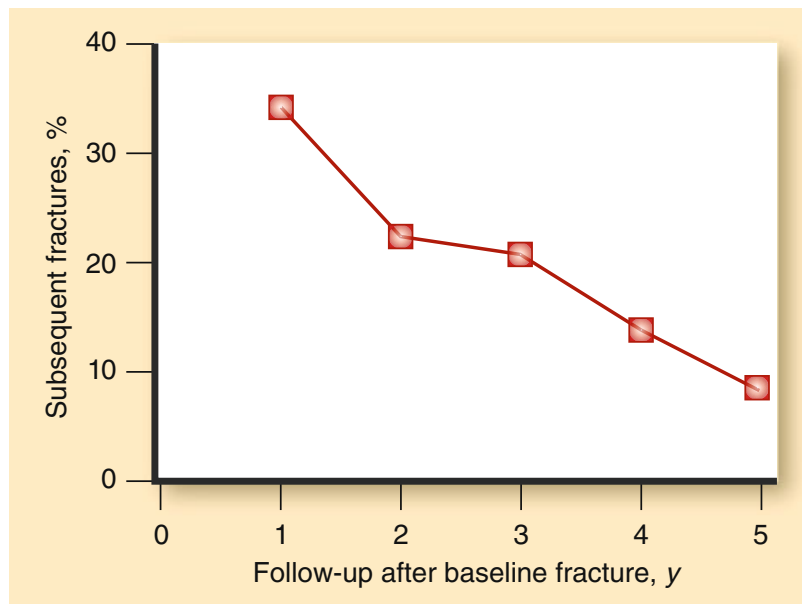

Fig. 3 Percentage of subsequent fractures during a 5-year follow-up period during a follow-up of 16 years. This clustering of fractures occurred regardless of the initial fracture location. However, with the expected increase in initial fracture risk with age, the excess risk decreased over time from initial fracture $(P=0.003$ and $P=0.001$ for women and men, respectively). After 10 years of follow-up, the subsequent absolute fracture risk was no longer elevated compared with the risk of a first fracture.

In a retrospective study, the time relation between fractures was studied between the time of prior fracture $(n>8,000)$ and the time a BMD measurement was performed, and the risk of subsequent fracture $(n>1,700)$ during a follow-up for a maximum of 10 years after the index BMD measurement [17]. The RR after major fractures was $1.9(95 \% \mathrm{CI}, 1.6-$ 2.3) after the BMD measurement in patients who had a fracture history less than 1 year before the BMD measurement, decreasing toward $1.6(95 \% \mathrm{CI}, 1.3-2.1)$ if the previous fracture had occurred more than 10 years ago $(P<$ 0.001 for trend). After minor fractures, the RR was 1.49 (95\% CI, 1.1-1.9) and 1.1 (95\% CI, 0.8-1.5), respectively [17]. However, this study did not report the exact timing between fractures, as the timing from BMD measurement and subsequent fracture was not reported. In view of the large database in that study, more detailed analysis of the time relation between fractures would be of interest.

The etiology of clustering of fractures needs further investigation in terms of bone-related risk factors (eg, cortical and trabecular bone loss due to immobility), fallrelated risk factors (eg, immobility, postural instability, muscle strength, plastering, use of walking device),and post-fracture care for morbidity (eg, locomotor revalidation after lower limb fractures).

\section{Conclusions}

The clustering of fractures in time indicates the need for immediate attention in both men and women who sustain a first fracture to prevent the occurrence of a subsequent fracture. The high increase of subsequent fractures immediately after a first fracture creates a window of opportunity to prevent subsequent fractures. However, no studies are available on fracture prevention immediately after a fracture. Such studies will be needed in view of the immediate burden of subsequent fractures in patients with a first fracture, who can easily be identified clinically.

In addition, when treatment with osteoporosis medication is considered, such treatment should be chosen on the basis of proven short-term efficacy in the reduction of vertebral and nonvertebral fractures. How far additional fall prevention strategies would further decrease subsequent fracture risk is unknown and is a field for further clinical research. 
Disclosure No potential conflicts of interest relevant to this article were reported.

Open Access This article is distributed under the terms of the Creative Commons Attribution Noncommercial License which permits any noncommercial use, distribution, and reproduction in any medium, provided the original author(s) and source are credited.

\section{References}

Papers of particular interest, published recently, have been highlighted as:

- Of importance

•- Of major importance

1. World Health Organization: W.H.O. Prevention and Management of Osteoporosis. Report of a WHO Scientific Group. WHO Technical Report Series 2003; No 921. Geneva, Switzerland: World Health Organization; 2003.

2. World Health Organization: WHO Scientific Group on the assessment of osteoporosis at primary health care level. Summary Meeting Report, Brussels, Belgium, 5-7 May 2004. Available at www.who.int/chp/topics/Osteoporosis.pdf. Accessed May 2010.

3. Klotzbuecher CM, Ross PD, Landsman PB, et al.: Patients with prior fractures have an increased risk of future fractures: a summary of the literature and statistical synthesis. J Bone Miner Res 2000, 15:721-727.

4. - Center JR, Bliuc D, Nguyen TV, Eisman JA: Risk of subsequent fracture after low-trauma fracture in men and women. JAMA 2007, 297:387-394. This study examined the subsequent risk of all fracture types, except fingers and toes, after an initial low-energy trauma fracture in men and women 60 years of age and over taking into account the time relation between fractures. The risk of subsequent fracture was highest immediately after the initial fracture, but was not higher than the risk for individuals without a fracture 10 years after the initial fracture $141 \%$ of the subsequent fractures occurred within 2 years).

5. ••van Geel TA, van Helden S, Geusens PP, et al.: Clinical subsequent fractures cluster in time after first fractures. Ann Rheum Dis 2009, 68:99-102. The results of this study extend the

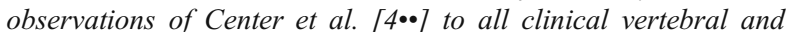
nonvertebral fractures, in women from menopause onward, and for both low- and high-energy trauma fractures. The initial fracture type was not a predictor for subsequent fractures. The subsequent fracture risk remained higher, compared with initial fracture risk, for 15 years after the initial fracture $(23 \%$ of the subsequent fractures occurred within 1 year).

6. Johnell O, Kanis JA, Oden A, et al.: Fracture risk following an osteoporotic fracture. Osteoporos Int 2004, 15:175-179.

7. Johnell O, Oden A, Caulin F, Kanis JA:. Acute and long-term increase in fracture risk after hospitalization for vertebral fracture. Osteoporos Int 2001, 12:207-214.

8. Lindsay RL, Silverman SL, Cooper C, et al.: Risk of new vertebral fracture in the year following a fracture. JAMA 2001, 285:320-323.

9. Harris ST, Watts NB, Genant HK, et al.: Effects of risedronate treatment on vertebral and nonvertebral fractures in women with postmenopausal osteoporosis: a randomized controlled trial. Vertebral Efficacy With Risedronate Therapy (VERT) Study Group. JAMA 1999, 282:1344-1352.

10. Reginster J, Minne HW, Sorensen $\mathrm{OH}$, et al.: Randomized trial of the effects of risedronate on vertebral fractures in women with established postmenopausal osteoporosis. Vertebral Efficacy with Risedronate Therapy (VERT) Study Group. Osteoporos Int 2000, 11:83-91.

11. McClung MR, Geusens P, Miller PD, et al.: Effect of risedronate on the risk of hip fracture in elderly women. Hip Intervention Program Study Group. N Engl J Med 2001, 344:333-340.

12. Friesendorff von M, Besjakov J, Akesson K: Long-term survival and fracture risk after hip fracture: a 22-year follow-up in women. J Bone Miner Res 2008, 23:1832-1841.

13. - Berry SD, Samelson EJ, Ngo L, et al.: Subsequent fracture in nursing home residents with a hip fracture: a competing risks approach. J Am Geriatr Soc 2008, 56:1887-1892. In long-term care residents with a surgically repaired hip fracture, 21\% sustained a subsequent fracture: $6 \%$ of residents experienced a subsequent fracture within 6 months, 12\% within 1 year, and 21\% within 5 years.

14. •• Ryg J, Rejnmark L, Overgaard S, et al.: Hip fracture patients at risk of second hip fracture: a nationwide population-based cohort study of 169,145 cases during 1977-2001. J Bone Miner Res 2009, 24:1299-1307. Approximately 170,000 patients with an initial hip fracture were included in this 25-year follow-up study. The cumulative incidence showed that $9 \%$ of the subsequent hip fractures were suffered within 1 year and 20\% within 5 years. The risk for a subsequent fracture remained significantly higher than the risk for individuals without a fracture for 15 years after the initial fracture.

15. Mitani S, Shimizu M, Abo M, et al.: Risk factors for second hip fractures among elderly patients. J Orthop Sci 2010, 15:192-197.

16. Lawrence TM, Wenn R, Boulton CT, Moran CG: Age-specific incidence of first and second fractures of the hip. J Bone Joint Surg Br 2010, 92:258-261.

17. Giangregorio L, Leslie W: Time since prior fracture is a risk modifier for ten year osteoporotic fractures. J Bone Miner Res 2010 Jan 29 [Epub ahead of print]. 